\title{
Microplankton growth, grazing, and community structure in the northern Gulf of Mexico
}

\author{
Suzanne L. Strom*, Michael W. Strom \\ Shannon Point Marine Center, Western Washington University, 1900 Shannon Point Road, Anacortes, Washington 98221 , USA
}

\begin{abstract}
Seawater dilution experiments were conducted during spring and fall in the continental shelf region of the northern Gulf of Mexico. Nutrient-enhanced phytoplankton growth rates of 0.7 to $2.2 \mathrm{~d}^{-1}$ were measured for the entire phytoplankton community; highest growth rates were associated with $>8 \mu \mathrm{m}$ cells. Phytoplankton growth was nutrient limited in all May experiments, and $>8 \mu \mathrm{m}$ phytoplankton, primarily diatoms, showed the strongest response to nutrient addition: their growth rates increased the most and reached the highest values. Rates of microzooplankton grazing on the entire phytoplankton community were moderate $\left(0\right.$ to $\left.0.7 \mathrm{~d}^{-1}\right)$. During a given experiment, patterns of grazing on 2 phytoplankton size fractions $(<8$ and $>8 \mu \mathrm{m})$ generally differed, and high rates of grazing $\left(>1 \mathrm{~d}^{-1}\right)$ on both $<8$ and $>8 \mu \mathrm{m}$ cells were sometimes observed. Across all experiments, grazing by microzooplankton averaged $30 \%$ of nutrient-enhanced phytoplankton growth. In May, when phytoplankton growth was strongly nutrient limited, grazing averaged $90 \%$ of natural (non-nutrient-enhanced) phytoplankton growth. These data indicate that microzooplankton can be a significant source of phytoplankton mortality, even in eutrophic coastal waters. The microzooplankton community, excluding cells $<5 \mu \mathrm{m}$, comprised primarily heterotrophic dinoflagellates and aloricate choreotrich ciliates. These organisms exhibited high net growth rates (mean $=0.8 \mathrm{~d}^{-1}$ ) during experiments at higher irradiance levels. Ingestion of chain diatoms by the dinoflagellate Gyrodinium sp. was observed in preserved samples; such grazing pathways, in which relatively large phytoplankton cells are consumed by protozoa, may be quantitatively important in this coastal ecosystem. Due to the variety of taxa and feeding mechanisms within the microzooplankton, their grazing impact was not restricted to the smallest phytoplankton cells, indicating that size-based models of trophic structure could yield misleading predictions about patterns of energy flow in this coastal ecosystem.
\end{abstract}

KEY WORDS: Microzooplankton $\cdot$ Phytoplankton $\cdot$ Protozoa $\cdot$ Grazing $\cdot$ Growth $\cdot$ Coastal

\section{INTRODUCTION}

The Mississippi River (USA) is the world's 6th largest in terms of freshwater discharge. Water flowing from the Mississippi is turbid and rich in nutrients: concentrations of nitrate and silicate at the river mouth can exceed $100 \mu \mathrm{M}$ (Turner \& Rabalais 1991). Rather than mixing with coastal waters in an estuary, approximately two-thirds of the Mississippi outflow discharges directly onto the outer continental shelf of the northem Gulf of Mexico. Flow of the river plume is predominantly to the southwest, although wind can sometimes drive low salinity waters to the east. An environment

\footnotetext{
•E-mail: stroms@henson.cc.wwu.edu
}

characterized by intense spatial variability is created as this large volume of riverine water mixes with the oligotrophic waters of the open Gulf.

Associated with the Mississippi outflow, particularly at intermediate salinities, are dense blooms of phytoplankton and other planktonic organisms (Lohrenz et al. 1990, Dagg \& Whitledge 1991, Chin-Leo \& Benner 1992). These organisms take up quantities of riverderived nutrients and dissolved organic matter. They may be deposited on the bottom, contributing to episodes of hypoxia in the plume region. Or they may be remineralized, with concomitant conversion of river-derived materials to other forms and dispersion to a wider oceanic region.

The increased availability of light as riverborne sediments are deposited in the delta region, coupled 
with high concentrations of nutrients, is probably the source of the intense phytoplankton blooms observed in the plume region (Riley 1937, Lohrenz et al. 1990). Another necessary condition for bloom formation, perhaps less widely recognized, is the absence of significant phytoplankton removal by grazers. Zooplankton grazers can structure phytoplankton communities by consuming some types of cells and not others. The remineralization of nitrogen, phosphorus, and silicon by grazers can influence nutrient availability to the phytoplankton. Fecal material and other waste products of zooplankton may be a component of material deposited to the sediments. Thus the activities of zooplanktonic grazers can influence when, where and how much material is deposited or remineralized in the Mississippi plume region.

Our goal in this study was to determine the effect of grazing by the microzooplankton $(<200 \mu \mathrm{m}$ phagotroph) community on phytoplankton in the continental shelf region of the northern Gulf of Mexico. We focused on the microzooplankton because it has recently been demonstrated that they can be important grazers in coastal ecosystems (Gifford 1988, Sherr et al. 1991, McManus \& Ederington-Cantrell 1992). Little, however, is known of their activities in relatively eutrophic waters, where phytoplankton populations often are dominated by large cells. Because protozoa, in particular, may have population growth rates as high as those of phytoplankton, the potential for these grazers to structure phytoplankton communities and to control blooms is high (Banse 1992). Our approach was to simultaneously determine the growth and the removal (by grazing) of various size fractions of phytoplankton, and to relate these processes to the composition and biomass of the microzooplankton community.

\section{MATERIALS AND METHODS}

Experiments were conducted in the northern Gulf of Mexico during October 1992 and May 1993 (Fig. 1). We used the dilution technique (Landry \& Hassett 1982) to estimate rates of phytoplankton growth and microzooplankton grazing. Seawater was collected several hours before dawn using a rosette of $10 \mathrm{l}$ Niskin bottles fitted with silicone closure tubing and O-rings. Filtered seawater was prepared by draining water from several of the Niskin bottles into a single 25 I polycarbonate carboy, then using a peristaltic pump to filter through a $0.2 \mu \mathrm{m}$ pore size cartridge filter (Nuclepore, October cruise; Gelman Versacap, May cruise) into 2.3 l polycarbonate incubation bottles. Filter cartridges were flushed with several liters of seawater before filtrate was collected. Unfiltered sea-

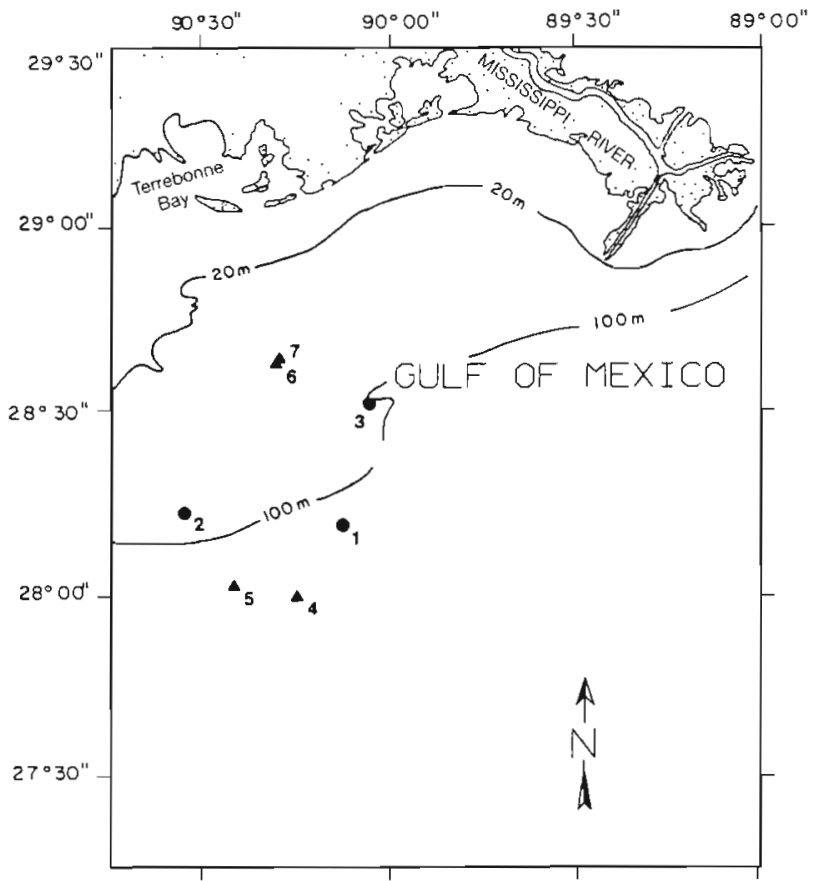

Fig. 1 Map of northern Gulf of Mexico showing station locations. (•) October 1992; («) May 1993

water was collected from the Niskin bottles in the same manner, except that tubing leading to the collection carboy had $200 \mu \mathrm{m}$ mesh Nitex screen fixed over the end to exclude macrozooplankton. Unfiltered seawater was then siphoned into incubation bottles in varying volumes to make up the dilution series. All tubing used was silicone; all containers were cleaned before and between experiments by soaking in $10 \%$ $\mathrm{HCl}$ and rinsing with Nanopure water.

Experiments were conducted with water from 2 depths (corresponding to 50 and $10 \%$ surface irradiance) on each sampling date. Irradiance profiles were generated using a Biospherical Instruments PNF-300 profiling irradiance sensor. During October, each dilution series consisted of duplicate bottles at each of 7 dilution levels; dilution levels (fraction unfiltered seawater) were nominally $0.07,0.15,0.25,0.40,0.65$, 0.80 , and 1.00. Nutrients were added to each bottle. In May, an additional pair of bottles at dilution level 1.00 was added to the series. This pair contained no added nutrients and provided a control for the effects of nutrient enrichment on phytoplankton growth. Nutrient enrichments were $5.0 \mu \mathrm{M} \mathrm{N}$ (as $\mathrm{NH}_{4} \mathrm{Cl}$ ) and $0.5 \mu \mathrm{MP}$ (as $\mathrm{KH}_{2} \mathrm{PO}_{4}$ ) at Stns 1, 2,4 and 5; $10 \mu \mathrm{MN}$ and $1 \mu \mathrm{M} P$ at Stns 3, 6 and 7 . Higher nutrient additions were used at stations nearer the river. Enrichment levels were chosen to allow at least 2 phytoplankton doublings in a day based on expected maximum phytoplankton standing stocks. 
A sample for determination of total initial chlorophyll concentration was taken from each bottle. Additional samples for determination of chlorophyll concentration in the $>8 \mu \mathrm{m}$ size fraction and planktonic community composition were taken from each undiluted bottle ( 2 depth $^{-1}$ in October, 4 depth $^{-1}$ in May). Bottles were then refilled completely using unfiltered seawater. Calculated initial concentrations were corrected for this refilling. Bottles were placed in bags of neutral density screen to simulate natural light levels and incubated in deck incubators cooled by flowing surface seawater (note that temperature differences between experimental depths were never $>2^{\circ} \mathrm{C}$ ).

After $24 \mathrm{~h}$ incubation, each bottle was subsampled in duplicate for total (GF/F filters) and $>8 \mu \mathrm{m}$ ( $8 \mu \mathrm{m}$ pore size polycarbonate filters) chlorophyll concentration. Chlorophyll concentration in the $<8 \mu \mathrm{m}$ size fraction was calculated by difference. Filters were frozen at $-20^{\circ} \mathrm{C}$ and chlorophyll content analyzed within 1 mo of sample collection (Welschmeyer et al. 1991). Bottles containing undiluted seawater were further subsampled for plankton community composition. Two types of samples were taken to characterize the planktonic community: ca $200 \mathrm{ml}$ was fixed in acid Lugol's (final concentration $12 \%$ ), while 50 or $100 \mathrm{ml}$ was fixed with ice-cold $10 \%$ glutaraldehyde (final concentration 0.5 to $1 \%$ ), DAPI-stained, filtered through a $1.0 \mu \mathrm{m}$ pore size polycarbonate filter over a $5.0 \mu \mathrm{m}$ pore size cellulose backing filter, slide-mounted, and frozen for later examination using epifluorescence microscopy (Sherr et al. 1992)

Phytoplankton growth $(\mu)$ and microzooplankton grazing $(g)$ rates were calculated from Model I regressions of apparent growth rate against dilution factor (Landry \& Hassett 1982) for total, $>8 \mu \mathrm{m}$, and $<8 \mu \mathrm{m}$ chlorophyll size fractions. The phytoplankton growth rate in control bottles with no added nutrients (May cruise only) was calculated as

$$
\mu_{(- \text {nutrients })}=\frac{1}{t} \ln \frac{P_{t}}{P_{0}}+g
$$

where $t$ is the incubation time (d), $P_{0}$ and $P_{t}$ are initial and final chlorophyll concentration in unenriched bottles, and $g$ is the grazing rate $\left(\mathrm{d}^{-1}\right)$ determined from the corresponding nutrient-enriched dilution series. Phytoplankton production was calculated from $\mu \times P_{m}$. where $P_{m}$ is the average chlorophyll concentration during the incubation:

$$
P_{m}=\frac{P_{0}\left[\mathrm{e}^{(\mu-g) t}-1\right]}{(\mu-g) t}
$$

(Frost 1972). An alternative method for calculating $\mu$ and $g$, the 3-point method (Gallegos 1989), was also used to account for possible grazing saturation. This method also minimizes biases due to growth of micro- zooplankton during incubations. Finally, a third estimate of $g$ values was derived from

$$
g=-\left[\frac{P_{t}}{P_{0}}-\mathrm{e}^{(\mu t)}\right]\left[\frac{\mu_{z}-\mu}{\mathrm{e}^{\left(\mu_{z} t\right)}-\mathrm{e}^{(\mu t)}}\right]
$$

(Gallegos 1989, his Eq. 11) where $\mu_{z}$ is net microzooplankton growth rate $\left(\mathrm{d}^{-1}\right)$ in undiluted bottles, and $\mu$ is calculated using the 3 -point method. This equation accounts for biases in estimates of $g$ due to microzooplankton growth under conditions of grazing saturation.

Lugol's-preserved samples taken at the beginning and end of each experiment from nutrient-enhanced bottles were analyzed using inverted microscopy to generate microzooplankton abundance and biomass estimates. Each cell was assigned to a taxonomic category and its dimensions measured using a digitizer connected to a microcomputer (Roff \& Hopcroft 1986). Linear dimensions were converted to cell volumes using standard geometric formulae. Conversion to cell

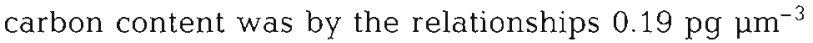
for ciliates (Putt \& Stoecker 1989); $0.14 \mathrm{pg} \mathrm{\mu m}^{-3}$ for dinoflagellates (Lessard 1991); $0.07 \mathrm{pg} \mathrm{m}^{-3}$ for copepod nauplii (Moloney \& Field 1991); and $\log \mathrm{C}=$ $-0.460+0.866(\log V)$ for sarcodines (Strathmann 1967). Epifluorescence microscopy was used to determine whether dinoflagellate taxa were autotrophic or heterotrophic (based on the presence or absence of chloroplasts). Strictly autotrophic taxa were not included in reported estimates, while taxa with both autotrophic and heterotrophic members (primarily Gymnodinium) were included in their entirety. Microzooplankton growth rates were calculated from initial and final abundance and cell $\mathrm{C}$ estimates, assuming exponential growth.

\section{RESULTS}

Water temperatures during the experiments ranged from 21.3 to $25.2^{\circ} \mathrm{C}$ and salinities from 31.9 to $36.3 \mathrm{ppt}$ (Table 1). Unfortunately, consistent nutrient data are not available for these cruises. Salinity levels, however, indicate a range of conditions from moderately river influenced (Stn 3) to fully oligotrophic (Stns 4 and 5). This observation is supported by measurements of the size structure of the phytoplankton community: at Stn 3, two-thirds to three-quarters of the chlorophyll was associated with cells $>8 \mu \mathrm{m}$, while at Stns 4 (both depths) and $5(10 \mathrm{~m})$ nearly all chlorophyll was in the $<8$ um size fraction (Table 1).

Rates of nutrient-enhanced phytoplankton growth ranged from 0.5 to $2.1 \mathrm{~d}^{-1}$ for total phytoplankton, with the highest rates observed during the October cruise (Table 2). At every station, growth rates were higher at 
Table 1. Environmental conditions and initial chlorophyll concentrations ( $\mu \mathrm{g}^{-1}$ ) for dilution experiments conducted during 2 cruises in the northern Gulf of Mexico

\begin{tabular}{|c|c|c|c|c|c|c|c|}
\hline \multirow[t]{2}{*}{ Stn } & \multirow[t]{2}{*}{ Date } & \multirow{2}{*}{$\begin{array}{l}\text { Depth } \\
\text { (m) }\end{array}$} & \multirow{2}{*}{$\begin{array}{l}\text { Temp. } \\
\left({ }^{\circ} \mathrm{C}\right)\end{array}$} & \multirow{2}{*}{$\begin{array}{l}\text { Salinity } \\
\text { (ppt) }\end{array}$} & \multicolumn{3}{|c|}{ Chlorophyll } \\
\hline & & & & & Total & $<8 \mu \mathrm{m}$ & $<8 \mu \mathrm{m} /$ Total \\
\hline \multirow[t]{2}{*}{1} & 20 Oct 1992 & 10 & 24.7 & 34.2 & 1.01 & 0.59 & 0.58 \\
\hline & & 25 & 25.2 & 35.4 & 0.37 & 0.25 & 0.68 \\
\hline \multirow[t]{2}{*}{2} & 22 Oct 1992 & 10 & 24.6 & 34.5 & 0.44 & 0.40 & 0.90 \\
\hline & & 25 & 24.6 & 34.5 & 0.11 & 0.09 & 0.83 \\
\hline \multirow[t]{2}{*}{3} & 26 Oct 1992 & 1 & 23.6 & 31.9 & 8.37 & 2.23 & 0.27 \\
\hline & & 3 & 24.0 & 32.0 & 8.01 & 2.80 & 0.35 \\
\hline \multirow[t]{2}{*}{4} & 6 May 1993 & 10 & 23.0 & 35.8 & 0.21 & 0.20 & 0.97 \\
\hline & & 50 & 21.3 & 36.3 & 0.43 & 0.41 & 0.96 \\
\hline \multirow[t]{2}{*}{5} & 8 May 1993 & 10 & 23.7 & 36.1 & 0.10 & 0.09 & 0.95 \\
\hline & & 50 & 21.7 & 36.2 & 0.33 & 0.26 & 0.79 \\
\hline \multirow[t]{2}{*}{6} & 10 May 1993 & 2 & 23.1 & 33.1 & 0.78 & 0.53 & 0.68 \\
\hline & & 20 & 21.3 & 35.7 & 0.80 & 0.61 & 0.77 \\
\hline \multirow[t]{2}{*}{7} & 12 May 1993 & 2 & 24.4 & 32.8 & 0.50 & 0.27 & 0.54 \\
\hline & & 20 & 22.0 & 36.1 & 0.71 & 0.59 & 0.83 \\
\hline
\end{tabular}

trations. While some dilution plots did show evidence of non-linearity (e.g Fig. 3B, $>8 \mu \mathrm{m}$ cells; Fig. 5B, total and $<8 \mu \mathrm{m}$ cells), there was no relationship between chlorophyll concentration and occurrence of possible grazing saturation during our experiments (Tables 1 \& 2).

In October, grazing was generally equivalent to $30-40 \%$ of nutrient-enhanced phytoplankton growth (Table 2). As actual (unenhanced) growth rates might have been lower, these percentages represent minimum estimates of grazing impact. In May, grazing was equivalent to $0-50 \%$ of unenhanced phytoplankton growth at offshore, more oligotrophic stations (4 and 5). Nearer to shore, phytoplankton growth was strongly nutrient limited and unenhanced growth rates were low $\left(0.1\right.$ to $\left.0.3 \mathrm{~d}^{-1}\right)$. At these stations ( 6 and 7 ), microzooplankton grazing rates were equivalent to $80-230 \%$ of phytoplankton growth.

Total microzooplankton abundance ranged from 5000 to 62000 cells $\mathrm{I}^{-1}$ and total biomass from 2.17 to $18.92 \mu \mathrm{g} \mathrm{C} \mathrm{l}^{-1}$ at the beginning of the experiments (Table 3). Biomass was highest at more eutrophic stations and, across all experiments, was closely correlated with total chlorophyll concentration ( $\mathrm{r}=0.918$, $\mathrm{p}<0.001, \mathrm{n}=14$ for $\log$ total chlorophyll versus $\log$

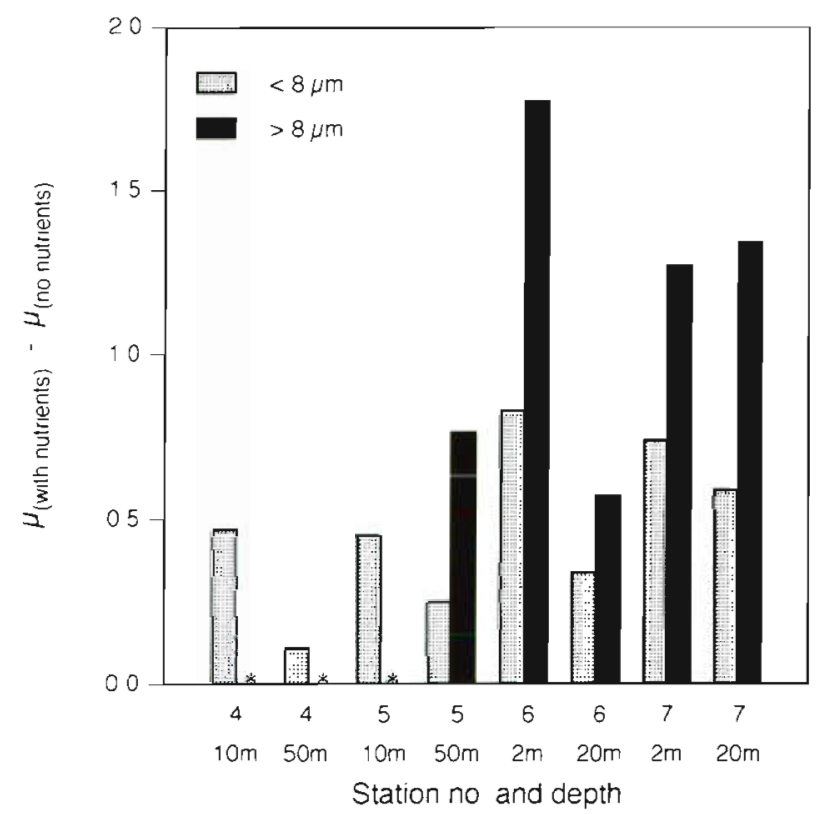

Fig. 2. Enhancement of growth rate $\left(\mathrm{d}^{-1}\right)$ by the addition of nutrients for phytoplankton in 2 size classes (May 1993). (*) Rates for $>8 \mu \mathrm{m}$ size class not determined ( $<5 \%$ of total chlorophyll in $>8 \mu \mathrm{m}$ size class during these experiments) 
Table 2. Rates of growth and grazing $\left(\mathrm{d}^{-1}\right)$ for $<8 \mu \mathrm{m},>8 \mu \mathrm{m}$, and total phytoplankton determined using the dilution technique (see text for methods of rate determination). Standard errors of rate estimates in parentheses. Ratios of $g: \mu$ derived from total chlorophyll regressions. Experiments showed evidence of grazing saturation; nd: not determined $(<5 \%$ of total chlorophyll in $>8 \mu \mathrm{m}$ size class at these stations)

\begin{tabular}{|c|c|c|c|c|c|c|c|c|c|c|c|c|}
\hline \multirow[t]{2}{*}{ Stn } & \multirow{2}{*}{$\begin{array}{l}\text { Depth } \\
(\mathrm{m})\end{array}$} & \multirow[t]{2}{*}{$\mathrm{n}$} & \multicolumn{3}{|c|}{ Growth (+ nutrients) } & \multicolumn{3}{|c|}{ Growth (- nutrients) } & \multicolumn{3}{|c|}{ Grazing } & \multirow[t]{2}{*}{$g: \mu$} \\
\hline & & & $<8 \mu \mathrm{m}$ & $>8 \mu \mathrm{m}$ & Total & $<8 \mu \mathrm{m}$ & $>8 \mu \mathrm{m}$ & Total & $<8 \mu \mathrm{m}$ & $>8 \mu \mathrm{m}$ & Total & \\
\hline \multirow[t]{2}{*}{1} & 10 & 14 & $\begin{array}{c}1.95 \\
(0.20)\end{array}$ & $\begin{array}{c}2.41 \\
(0.05)\end{array}$ & $\begin{array}{c}2.06 \\
(0.04)\end{array}$ & & & & $\begin{array}{l}2.34 \\
(0.32)\end{array}$ & $\begin{array}{c}0.16 \\
(0.09)\end{array}$ & $\begin{array}{c}0.58 \\
(0.07)\end{array}$ & 0.3 \\
\hline & 25 & 14 & $\begin{array}{c}0.70 \\
(0.03)\end{array}$ & $\begin{array}{r}1.30^{\circ} \\
10.151\end{array}$ & $\begin{array}{c}0.95 \\
(0.08)\end{array}$ & & & & $\begin{array}{c}-0.03 \\
(0.25)\end{array}$ & $\begin{array}{l}1.07^{\circ} \\
(0.24)\end{array}$ & $\begin{array}{c}0.36 \\
\{0.14\}\end{array}$ & 0.4 \\
\hline \multirow[t]{2}{*}{2} & 10 & 12 & $\begin{array}{c}0.95 \\
(0.12\}\end{array}$ & $\begin{array}{c}1.64 \\
(0.17)\end{array}$ & $\begin{array}{c}1.04 \\
(0.11)\end{array}$ & & & & $\begin{array}{c}0.35 \\
(0.17)\end{array}$ & $\begin{array}{c}0.34 \\
(0.24)\end{array}$ & $\begin{array}{c}0.35 \\
(0.16)\end{array}$ & 0.3 \\
\hline & 25 & 12 & $\begin{array}{c}0.45 \\
(0.12)\end{array}$ & $\begin{array}{r}1.43^{\circ} \\
(0.15)\end{array}$ & $\begin{array}{c}0.71^{\circ} \\
(0.12)\end{array}$ & & & & $\begin{array}{c}0.08 \\
(0.18)\end{array}$ & $\begin{array}{c}0.38^{\circ} \\
(0.22)\end{array}$ & $\begin{array}{r}0.17^{\circ} \\
(0.17)\end{array}$ & 0.3 \\
\hline \multirow[t]{2}{*}{3} & 1 & 14 & $\begin{array}{c}2.20 \\
(0.12)\end{array}$ & $\begin{array}{c}2.37 \\
(0.12)\end{array}$ & $\begin{array}{c}2.22 \\
(0.06)\end{array}$ & & & & $\begin{array}{c}-0.28 \\
(0.19)\end{array}$ & $\begin{array}{c}1.73 \\
(0.21)\end{array}$ & $\begin{array}{c}0.67 \\
(0.10)\end{array}$ & 0.3 \\
\hline & 3 & 12 & $\begin{array}{c}0.95 \\
(0.15)\end{array}$ & $\begin{array}{c}0.56 \\
(0.12)\end{array}$ & $\begin{array}{c}0.68 \\
(0.10)\end{array}$ & & & & $\begin{array}{c}0.49 \\
(0.23)\end{array}$ & $\begin{array}{c}-0.33 \\
(0.18)\end{array}$ & $\begin{array}{c}-0.08 \\
(0.15)\end{array}$ & - \\
\hline \multirow[t]{2}{*}{4} & 10 & 14 & $\begin{array}{c}0.92 \\
(0.05)\end{array}$ & nd & $\begin{array}{c}0.90 \\
(0.05)\end{array}$ & 0.52 & nd & 0.46 & $\begin{array}{c}0.22 \\
(0.09)\end{array}$ & nd & $\begin{array}{c}0.15 \\
(0.09)\end{array}$ & 0.3 \\
\hline & 50 & 14 & $\begin{array}{c}0.53 \\
(0.05)\end{array}$ & nd & $\begin{array}{c}0.53 \\
(0.05)\end{array}$ & 0.42 & nd & 0.42 & $\begin{array}{c}0.18 \\
(0.08)\end{array}$ & nd & $\begin{array}{c}0.18 \\
(0.08)\end{array}$ & 0.4 \\
\hline \multirow[t]{2}{*}{5} & 10 & 14 & $\begin{array}{c}1.11 \\
(0.06)\end{array}$ & nd & $\begin{array}{c}1.11 \\
(0.06)\end{array}$ & 0.66 & nd & 0.66 & $\begin{array}{c}-0.07 \\
(0.10)\end{array}$ & nd & $\begin{array}{c}-0.10 \\
(0.10)\end{array}$ & - \\
\hline & 50 & 14 & $\begin{array}{c}0.82 \\
(0.07)\end{array}$ & $\begin{array}{c}1.64 \\
(0.05)\end{array}$ & $\begin{array}{c}1.06 \\
(0.06)\end{array}$ & 0.57 & 0.88 & 0.66 & $\begin{array}{c}0.27 \\
\{0.13\}\end{array}$ & $\begin{array}{c}0.38 \\
(0.10)\end{array}$ & $\begin{array}{c}0.31 \\
(0.10)\end{array}$ & 0.5 \\
\hline \multirow[t]{2}{*}{6} & 2 & 14 & $\begin{array}{c}1.12 \\
(0.07)\end{array}$ & $\begin{array}{c}1.90 \\
(0.08)\end{array}$ & $\begin{array}{c}1.41 \\
(0.03)\end{array}$ & 0.29 & 0.13 & 0.12 & $\begin{array}{c}0.47 \\
(0.11)\end{array}$ & $\begin{array}{c}0.20 \\
(0.12)\end{array}$ & $\begin{array}{c}0.27 \\
(0.05)\end{array}$ & 2.3 \\
\hline & 20 & 14 & $\begin{array}{c}0.71^{\circ} \\
(0.08)\end{array}$ & $\begin{array}{c}0.61 \\
(0.05)\end{array}$ & $\begin{array}{c}0.69^{\circ} \\
(0.07)\end{array}$ & 0.37 & 0.04 & 0.30 & $\begin{array}{c}0.31^{\circ} \\
(0.13)^{\circ}\end{array}$ & $\begin{array}{c}0.05 \\
(0.08)\end{array}$ & $\begin{array}{r}0.25^{\circ} \\
(0.11)\end{array}$ & 0.8 \\
\hline \multirow[t]{2}{*}{7} & 2 & 14 & $\begin{array}{c}1.30 \\
(0.05)\end{array}$ & $\begin{array}{c}1.03 \\
(0.04)\end{array}$ & $\begin{array}{l}1.19 \\
(0.05)\end{array}$ & 0.56 & -0.24 & 0.28 & $\begin{array}{c}0.32 \\
(0.08)\end{array}$ & $\begin{array}{c}0.16 \\
(0.06)\end{array}$ & $\begin{array}{c}0.25 \\
(0.07)\end{array}$ & 0.9 \\
\hline & 20 & 14 & $\begin{array}{l}0.95 \\
(0.03)\end{array}$ & $\begin{array}{c}1.39 \\
(0.06)\end{array}$ & $\begin{array}{l}1.04 \\
(0.04)\end{array}$ & 0.36 & 0.05 & 0.28 & $\begin{array}{c}0.61 \\
(0.06)\end{array}$ & $\begin{array}{c}0.35 \\
(0.10)\end{array}$ & $\begin{array}{c}0.54 \\
(0.06)\end{array}$ & 1.9 \\
\hline
\end{tabular}

Table 3. Total microzooplankton abundance (cells $\left.\mathrm{l}^{-1}\right)$, cell volume $\left(\mu \mathrm{m}^{3} \mathrm{l}^{-1} \times 10^{8}\right)$, and biomass ( $\mu \mathrm{g} \mathrm{C}^{-1}$ ) in northern Gulf of Mexico waters. n: cells counted and measured in each sample

\begin{tabular}{|ccccccl|}
\hline Stn & $\begin{array}{c}\text { Depth } \\
(\mathrm{m})\end{array}$ & $\mathrm{n}$ & \multicolumn{5}{c|}{$\begin{array}{l}\text { Total microzooplankton } \\
\text { Abundance Cell volume Cell C }\end{array}$} \\
\hline 1 & 10 & 301 & 16000 & 0.31 & 4.53 & Many diatoms, dominant genera Pseudonitzschia, Skeletonema \\
& 25 & 155 & 9100 & 1.58 & 4.67 & Detritus, scuticociliates \\
2 & 10 & 266 & 19600 & 0.98 & 3.13 & \\
& 25 & 198 & 13700 & 1.41 & 2.29 & \\
3 & 1 & 299 & 62000 & 10.00 & 18.92 & Very many diatoms, dominant genera Pseudonitzschia, Skeletonema \\
& 3 & 267 & 53000 & 10.32 & 13.69 & Same as at 1 m \\
4 & 10 & 967 & 10700 & 0.23 & 2.17 & Blue water, Sargassum \\
& 50 & 352 & 8300 & 0.45 & 2.93 & \\
5 & 10 & 266 & 4800 & 0.20 & 2.68 & Blue water, Sargassum \\
& 50 & 440 & 9700 & 0.43 & 4.54 & \\
6 & 2 & 365 & 19900 & 1.88 & 5.41 & Trichodesmium bloom \\
7 & 20 & 310 & 32900 & 3.27 & 8.58 & No Trichodesmium present \\
& 2 & 471 & 24200 & 1.68 & 5.29 & Dense population of doliolid Thalia democratica present 11 May \\
& 20 & 325 & 31700 & 1.16 & 6.44 & \\
\hline
\end{tabular}



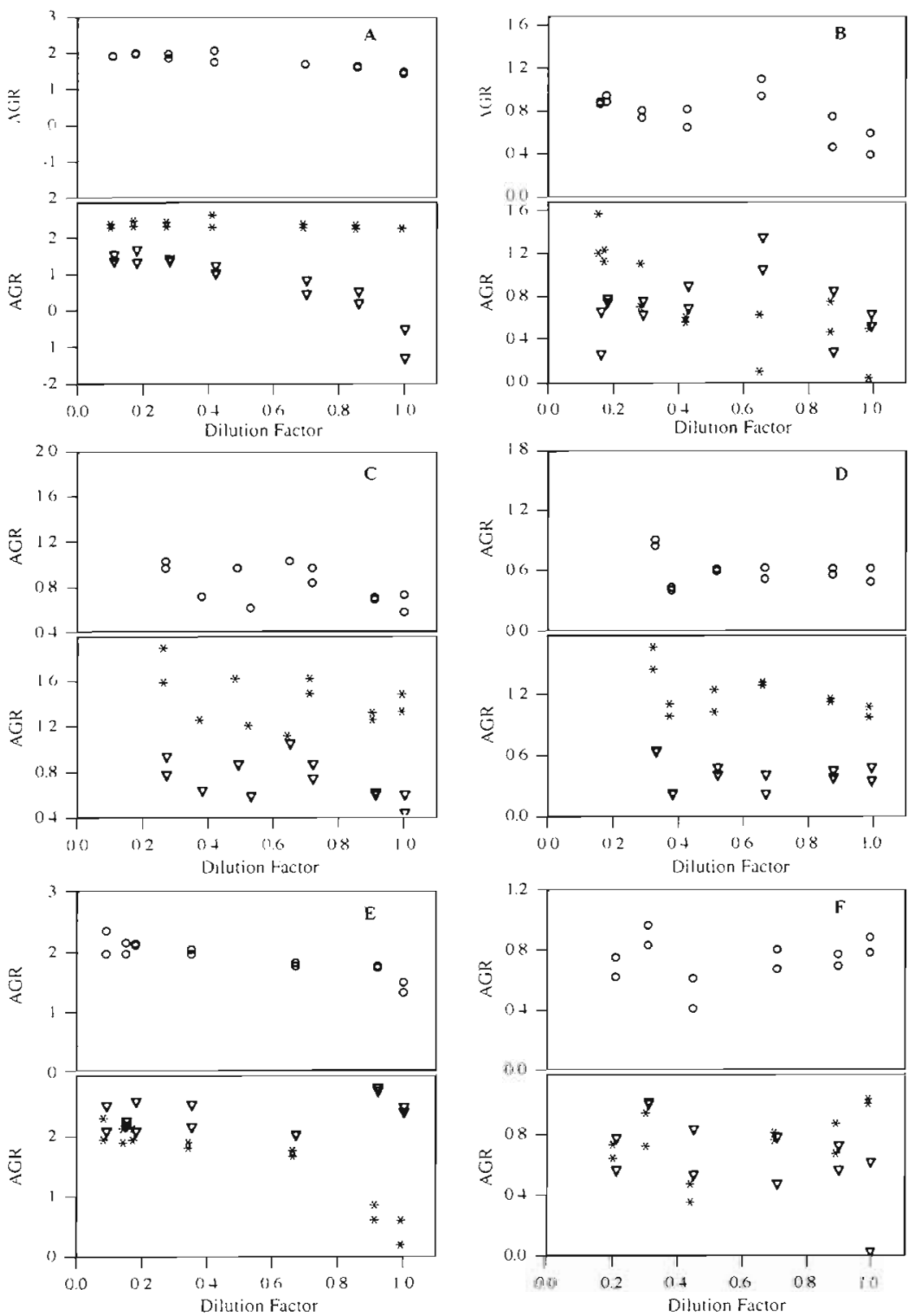

Fig. 3. Dilution plots (apparent growth rate versus dilution factor) for October experiments showing total chlorophyll $(O)$ and chlorophyll in $<8 \mu \mathrm{m}$ cells ( $\nabla)$ and in $>8 \mu \mathrm{m}$ cells (*). (A) Stn 1, $10 \mathrm{~m}$; (B) Stn 1, $25 \mathrm{~m}$; (C) Stn 2, $10 \mathrm{~m}$; (D) $\operatorname{Stn} 2,25 \mathrm{~m}$. (E) $\operatorname{Stn} 3,1 \mathrm{~m}$; (F) $\operatorname{Stn} 3,3 \mathrm{~m}$

microzooplankton biomass). The microzooplankton community was composed primarily of choreotrich ciliates and dinoflagellates (Fig. 6); few sarcodines or copepod nauplii were observed. Note that organisms $<5 \mu \mathrm{m}$ in size were not enumerated. Thus the smallest nanoflagellates, which are probably important as grazers of Synechococcus and picoeukaryotes, were not included in microzooplankton biomass estimates.

In general, microzooplankton biomass was approximately equally divided between ciliates and dinoflagellates (Fig. 6). At Stn 3, however, dinoflagellates dominated the biomass, while at $\operatorname{Stn} 5(10 \mathrm{~m})$, ciliates were dominant. The major dinoflagellate groups were Gyrodinium, Gymnodinium, and thecate Protoperidiniumand Oblea-like forms. Ciliates were mainly aloricate choreotrichs. Few tintinnids were observed, but scuticociliates were abundant in some of the deeper samples (Stn 1, $25 \mathrm{~m}_{\mathrm{i}}$ Stn 4, $50 \mathrm{~m}$ ). Heterotrophic protists with cell volumes of 200 to $2000 \mu^{3}$ were the most abundant overall. The distribution of cell volume varied considerably between stations, with oligotrophic waters tending to have the smallest organisms and more eutrophic waters the largest (Fig. 7).

Dinoflagellates of the genus Gyrodinium may have been important consumers of large diatoms during some of these experiments. In preserved samples, we observed Gyrodinium in various stages of engulfing chain diatoms (Fig. 8). This mode of feeding was observed primarily in samples from $1 \mathrm{~m}$ depth at Stn 3, where a high rate of grazing on large phytoplankton $\left(1.7 \mathrm{~d}^{-1}\right)$ was measured.

Cell-specific net growth rates of microzooplankton during the experiments ranged from -0.6 to $1.4 \mathrm{~d}^{-1}$. while carbon-specific net growth rates ranged from -0.6 to $1.7 \mathrm{~d}^{-1}$ (Table 4 ). Most rates were $>0$ and several were $>0.7 \mathrm{~d}^{-1}$. There were no significant correlations between $\mathrm{C}$-specific microzooplankton growth rates and phytoplankton biomass, growth rate, or production, either total or by size class. Discrepancies between celland $\mathrm{C}$-specific growth rates indicate that average cell sizes of microzooplankton changed during the experiments. For all October experiments and at Stns 6 and 7 in May, ciliate, heterotrophic dinoflagellate (hdino), and total microzooplankton growth data indicate an increase in cell volume of individual protists over the incubation period. At Stns 4 and 5 in May, C-specific growth rates were generally less than cell-specific growth rates, such that individual protist cell volumes decreased over the incubation period.

Mean C-specific net growth rates for total microzooplankton at high irradiance levels $\left(0.8 \mathrm{~d}^{-1}\right)$ were significantly higher than net growth rates at low irradiance levels $\left(0.2 \mathrm{~d}^{-1}\right.$ ) (Students paired t-test, $\mathrm{n}=14$, 

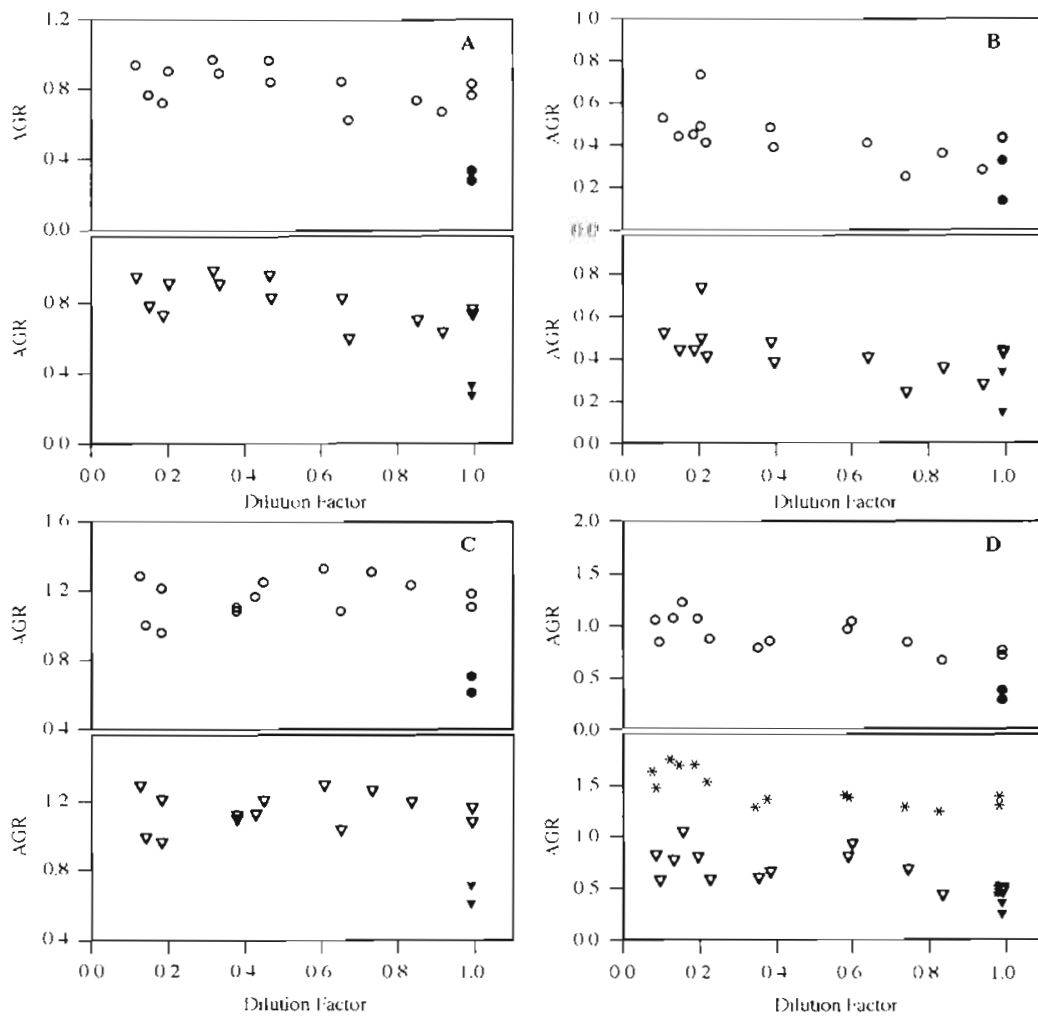

Fig. 4. Dilution plots (apparent growth rate vs dilution factor) for May experiments. Symbols as in Fig. 3. Solid symbols indicate treatments with no added nutrients. (A) $\operatorname{Stn} 4,10 \mathrm{~m}$; (B) $\operatorname{Stn} 4,50 \mathrm{~m}$; (C) $\operatorname{Stn} 5,10 \mathrm{~m}$; (D) $\operatorname{Stn} 5,50 \mathrm{~m}$ nity. As dilution reduces food availability, intrinsic growth and predation may not remain constant along the dilution gradient. Non-linearities are introduced when initial, undiluted food concentrations are above levels at which grazing responses saturate.

Because there was evidence of saturated grazing in several of our experiments, we used the 3-point method (Gallegos 1989) to derive a set of alternate estimates of phytoplankton $\mu$ (Table 5). The 3-point estimate of $\mu$ relies only on apparent growth in the 2 most dilute treatments, thus avoiding biases due to saturated grazing at higher phytoplankton concentrations. Use of the 3-point method should also minimize biases due to microzooplankton growth in systems where 'undiluted' microzooplankton growth rates are high (Gallegos 1989). The 3 -point estimates of $\mu$ were within $20 \%$ of estimates based on linear regression in half of our experiments. In the other half, 3-point estimates of $\mu$ ranged up to twice the values derived from linear regression (Table 5). $0.01<\mathrm{p}<0.05$ ), in parallel with the trend for phytoplankton growth rates. There were no consistent differences between growth rates of ciliates and hdinos, and growth rates averaged over all experiments were remarkably similar for these 2 protist groups (Table 4). Initial and final ratios of choreotrich ciliates:hdinos (Table 4) indicate that shifts in gross taxonomic composition occurred during some experiments. There were, however, no obvious trends (e.g. toward increased dominance by one or the other taxon) apparent in these data.

Significant changes in microzooplankton density during incubations complicate interpretation of dilution experiment results. Accumulation (net growth) of microzooplankton in any given bottle will be the sum of increases due to intrinsic growth and losses due to death or predation from within the microzooplankton commu-
Table 4. Cell- and carbon-specific net growth rates $\left(d^{-1}\right)$ and biomass ratios (initial and final) for choreotrich ciliates, heterotrophic dinoflagellates (Hdino), and total microzooplankton in undiluted, nutrient-enriched bottles. Ciliate and dinoflagellate carbon contents were determined using linear conversions from cell volume (see 'Methods')

\begin{tabular}{|c|c|c|c|c|c|c|c|c|c|}
\hline \multirow[t]{2}{*}{ Str } & \multirow{2}{*}{$\begin{array}{l}\text { Depth } \\
\text { (m) }\end{array}$} & \multicolumn{3}{|c|}{ Cell-specific } & \multicolumn{3}{|c|}{ Carbon-specific } & \multicolumn{2}{|c|}{ Ciliate:Hdino } \\
\hline & & Ciliate & Hdino & Total & Ciliate & Hdino & Total & Initial & Final \\
\hline \multirow[t]{2}{*}{1} & 10 & 0.15 & 0.65 & 0.37 & 0.37 & 0.93 & 0.59 & 2.02 & 1.15 \\
\hline & 25 & 0.18 & -0.16 & -0.21 & 0.32 & 0.13 & 0.13 & 1.23 & 1.47 \\
\hline \multirow[t]{2}{*}{2} & 10 & 0.09 & 0.91 & 0.33 & 0.60 & 0.82 & 0.67 & 1.84 & 1.49 \\
\hline & 25 & 0.07 & 0.49 & 0.20 & 0.64 & 0.56 & 0.58 & 1.22 & 1.31 \\
\hline \multirow[t]{2}{*}{3} & 1 & 0.52 & 0.40 & 0.46 & 0.91 & 0.87 & 0.89 & 0.66 & 0.70 \\
\hline & 3 & 0.15 & 0.16 & 0.16 & 1.11 & 0.80 & 0.92 & 0.46 & 0.63 \\
\hline \multirow[t]{2}{*}{4} & 10 & 0.74 & 0.43 & 0.52 & 0.49 & 0.83 & 0.68 & 1.14 & 0.81 \\
\hline & 50 & 1.42 & 0.73 & 0.95 & 0.81 & -0.18 & 0.24 & 0.79 & 2.12 \\
\hline \multirow[t]{2}{*}{5} & 10 & 1.17 & 0.56 & 0.83 & -0.02 & 0.78 & 0.18 & 4.67 & 2.10 \\
\hline & 50 & -0.22 & 0.36 & 0.07 & -0.97 & -0.31 & -0.63 & 1.07 & 0.56 \\
\hline \multirow[t]{2}{*}{6} & 2 & 1.47 & 1.29 & 1.38 & 2.00 & 1.26 & 1.66 & 0.84 & 1.76 \\
\hline & 20 & -0.62 & -0.64 & -0.63 & -0.21 & -0.23 & -0.23 & 0.61 & 0.62 \\
\hline \multirow[t]{4}{*}{7} & 2 & 0.51 & 0.12 & 0.28 & 1.05 & 0.32 & 0.76 & 1.16 & 2.43 \\
\hline & 20 & -0.15 & -0.33 & -0.23 & -0.08 & 0.03 & 0.07 & 1.33 & 1.19 \\
\hline & Mean & 0.39 & 0.35 & 0.32 & 0.50 & 0.47 & 0.47 & & \\
\hline & $\mathrm{SE}$ & 0.17 & 0.13 & 0.14 & 0.19 & 0.13 & 0.15 & & \\
\hline
\end{tabular}


Table 5. Comparison of dilution experiment rate estimates $\left(\mathrm{d}^{-1}\right)$ obtained using different computation methods. Only results for the total phytoplankton community are shown. l.r.: estimates obtained from Model I linear regression of apparent growth rate on dilution factor; 3-pt.: estimates obtained using the 3-point method (Gallegos 1989); mz: microzooplankton net growth rates incorporated into grazing estimates (Eq. 3; Gallegos 1989)

\begin{tabular}{|cccccrrr|}
\hline \multirow{2}{*}{ Stn } & $\begin{array}{c}\text { Depth } \\
(\mathrm{m})\end{array}$ & \multicolumn{2}{c}{ Growth $(\mu)$} & \multicolumn{3}{c|}{ Grazing $(g)$} \\
& & l.r. & 3-pt. & 1.r. & 3-pt. & \multicolumn{1}{c|}{$\mathrm{mz}$} \\
\hline 1 & 10 & 2.06 & 1.81 & 0.58 & 0.40 & 0.57 \\
& 25 & 0.95 & 0.60 & 0.36 & 0.12 & 0.14 \\
2 & 10 & 1.04 & 2.23 & 0.35 & 1.58 & 1.57 \\
& 25 & 0.71 & 1.82 & 0.17 & 1.28 & 1.26 \\
3 & 1 & 2.22 & 2.32 & 0.67 & 0.91 & 1.12 \\
& 3 & 0.68 & 0.21 & -0.08 & -0.62 & -0.59 \\
4 & 10 & 0.90 & 0.94 & 0.15 & 0.14 & 0.15 \\
& 50 & 0.53 & 0.51 & 0.18 & 0.08 & 0.08 \\
5 & 10 & 1.11 & 1.30 & -0.10 & 0.15 & 0.23 \\
& 50 & 1.06 & 0.61 & 0.31 & -0.14 & -0.27 \\
6 & 2 & 1.41 & 1.35 & 0.27 & 0.19 & 0.15 \\
& 20 & 0.69 & 1.07 & 0.25 & 0.70 & 0.90 \\
7 & 2 & 1.19 & 1.04 & 0.25 & 0.14 & 0.15 \\
& 20 & 1.04 & 1.37 & 0.54 & 0.90 & 1.06 \\
& & & & & & \\
\hline
\end{tabular}

When undiluted phytoplankton concentrations are below levels resulting in grazing saturation, biases in estimates of $g$ due to changes in microzooplankton density during experiments can be dealt with by regressing apparent growth rate against mean grazer density rather than fraction unfiltered seawater (Landry et al. 1984, Gallegos 1989). Under conditions of saturated grazing, one can assume that microzooplankton growth is independent of dilution and $g$ can be estimated from measured microzooplankton growth rates and a 3-point estimate of $\mu$ (Eq. 3). Alternatively, one can provide some independent estimate of the effect of dilution on $g$ (e.g. disappearance of fluorescent particles), and use that estimate as the independent variable in the regression approach (Landry et al. 1995). In the absence of either measurements of mean grazer density in all incubation bottles or an independent estimate of $g$, we used the 3-point method and Eq. (3) to bracket estimates of $g$. Both methods use only highly dilute and undiluted treatments to estimate $g$; Eq. (3) further accounts for growth of microzooplankton during the incubations. Estimates of $g$ by these 2 methods were similar (Table 5). Discrepancies between these and the linear-regressionbased estimates of $g$ appeared to be due largely to differences in estimates of $\mu$.

\section{DISCUSSION}

The grazing impact of the microzooplankton community has been found to be significant in a number of nearshore environments (e.g. Burkill et al. 1987, Paranjape 1987, Gifford 1988, McManus \& Ederington-Cantrell 1992), including the coastal Gulf of Mexico (Dagg 1995, Fahnenstiel et al. 1995). Supported by this growing body of evidence, concepts of trophic structure in nearshore planktonic food webs are beginning to extend beyond the diatom-copepod-fish paradigm (e.g. Jackson \& Eldridge 1992, Biscaye et al. 1994). Our data support the idea that microzooplankton grazing can be a significant source of mortality for phytoplankton populations in nearshore waters: we measured grazing rates of $>1 \mathrm{~d}^{-1}$ in several instances. Further, findings reported here support the notion that size-based models of food web relationships may be of limited predictive value (Longhurst
Fig. 5. Dilution plots (apparent growth rate vs dilution factor) for May experiments. Symbols as in Fig. 3. Solid symbols indicate treatments with no added nutrients. (A) $\operatorname{Stn} 6,2 \mathrm{~m}$; (B) $\operatorname{Stn} 6,20 \mathrm{~m}$; (C) $\operatorname{Stn} 7,2 \mathrm{~m}$; (D) $\operatorname{Stn} 7,20 \mathrm{~m}$ 
1991). Based on the idea that microzooplankton are most important as grazers of the smallest phytoplankton cells, one might have predicted the highest importance of microzooplankton grazing at the most oligotrophic stations in this study. Such was not the case. The highest absolute grazing rates were measured at stations (1 and 3) with relatively low salinities (i.e. a moderate riverine influence) and high concentrations of chlorophyll in large cells. Highest grazing rates relative to phytoplankton growth were at stations (6 and 7) where phytoplankton growth was strongly nutrient limited, but where chlorophyll levels were higher than at the most oligotrophic stations (4 and 5).

High rates of phytoplankton growth were observed during most of our experiments. The highest rates were associated with near-surface waters at Stns 1 and 3, where large diatoms were abundant. Diatoms as a group are known to have high potential growth rates relative to other microalgae (Banse 1982, Furnas 1990) and this was borne out by our data. Large phytoplankton also showed the strongest response to nutrient addition in our experiments: their growth rates increased more and reached higher levels than did growth rates of small phytoplankton. Diatom blooms are a characteristic feature of continental shelf ecosystems and the high potential growth rates of these cells, coupled with the capacity for large growth rate increases in response to nutrient inputs, are a key part of the explanation.

Processes that remove diatoms from euphotic zone waters are the other key regulators of bloom formation. Loss of diatoms from the upper water column is typically attributed to sinking and grazing by mesozooplankton. Our data indicate that microzooplankton grazing can contribute to removal of diatoms from surface waters under some conditions. At $1 \mathrm{~m}$ depth at Stn 3, a site dominated by the diatom genera Pseudonitzschia and Skeletonema, microzooplankton grazing $\left(1.7 \mathrm{~d}^{-1}\right)$ was nearly equivalent to nutrient-enhanced growth of large phytoplankton $\left(2.4 \mathrm{~d}^{-1}\right)$. Hdinos known to graze on diatoms, including Gyrodinium (Bursa 1961, Hansen 1992), Protoperidinium, and Oblea (Jacobson \& Anderson 1986, Strom \& Buskey 1993), were abundant at this site (Figs. 6 \& 8).
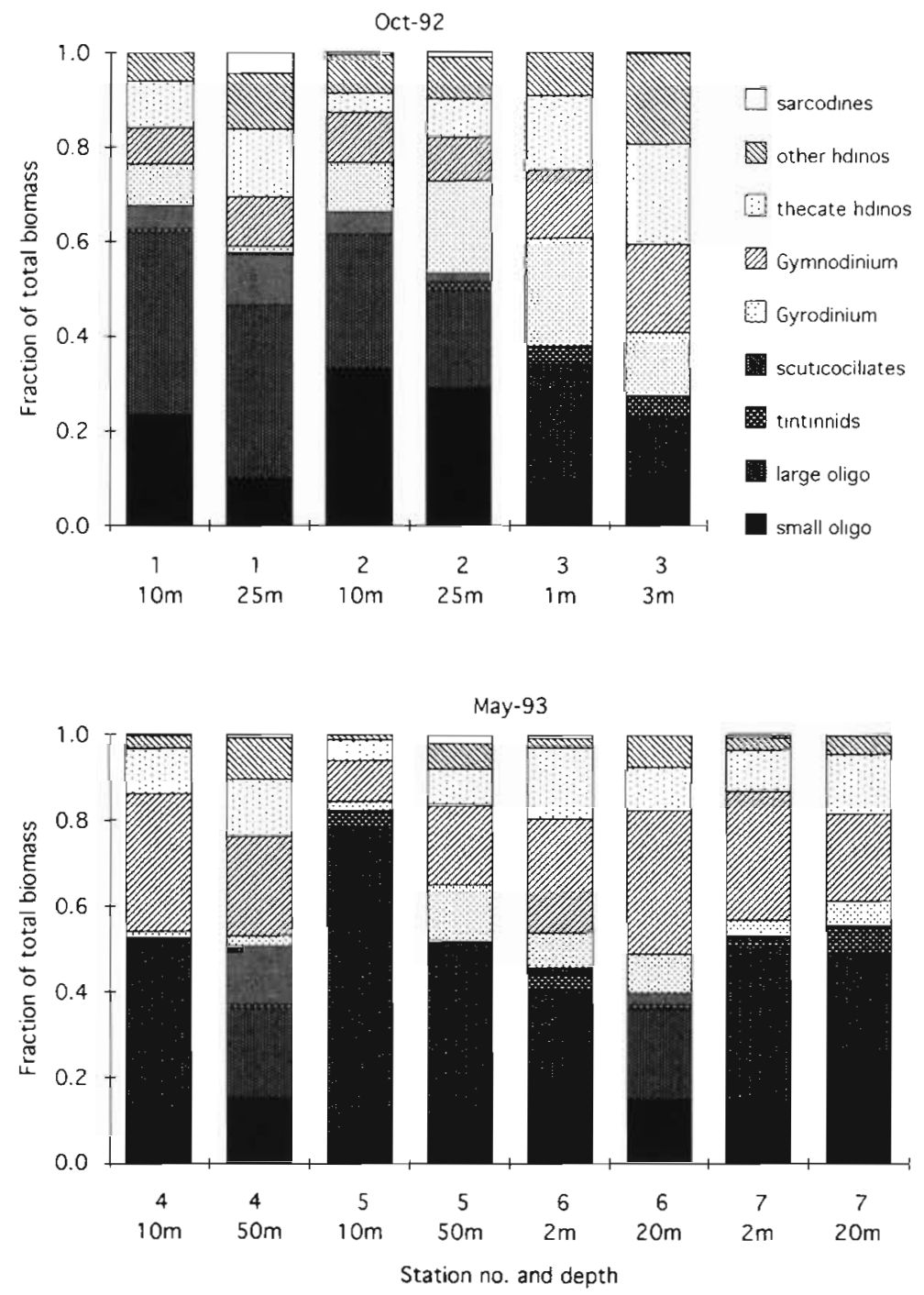

Fig. 6. Initial microzooplankton community composition for dilution experiments conducted in October 1992 and May 1993. hdinos: heterotrophic dinoflagellates; oligo: oligotrichs (aloricate choreotrich ciliates)
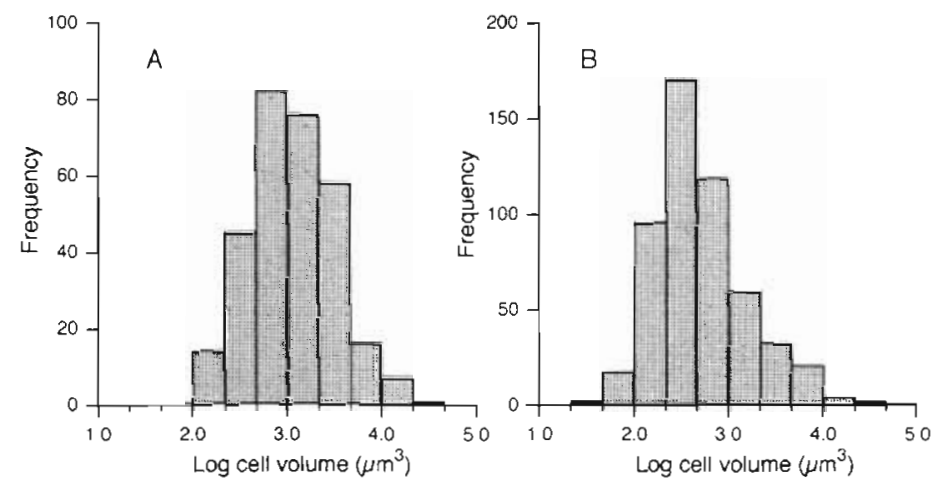

Fig. 7. Frequency distribution of microzooplankton cell volume at 2 contrasting stations. (A) Stn 3, $1 \mathrm{~m}$ (October)i (B) Stn 4, $10 \mathrm{~m}$ (May) 

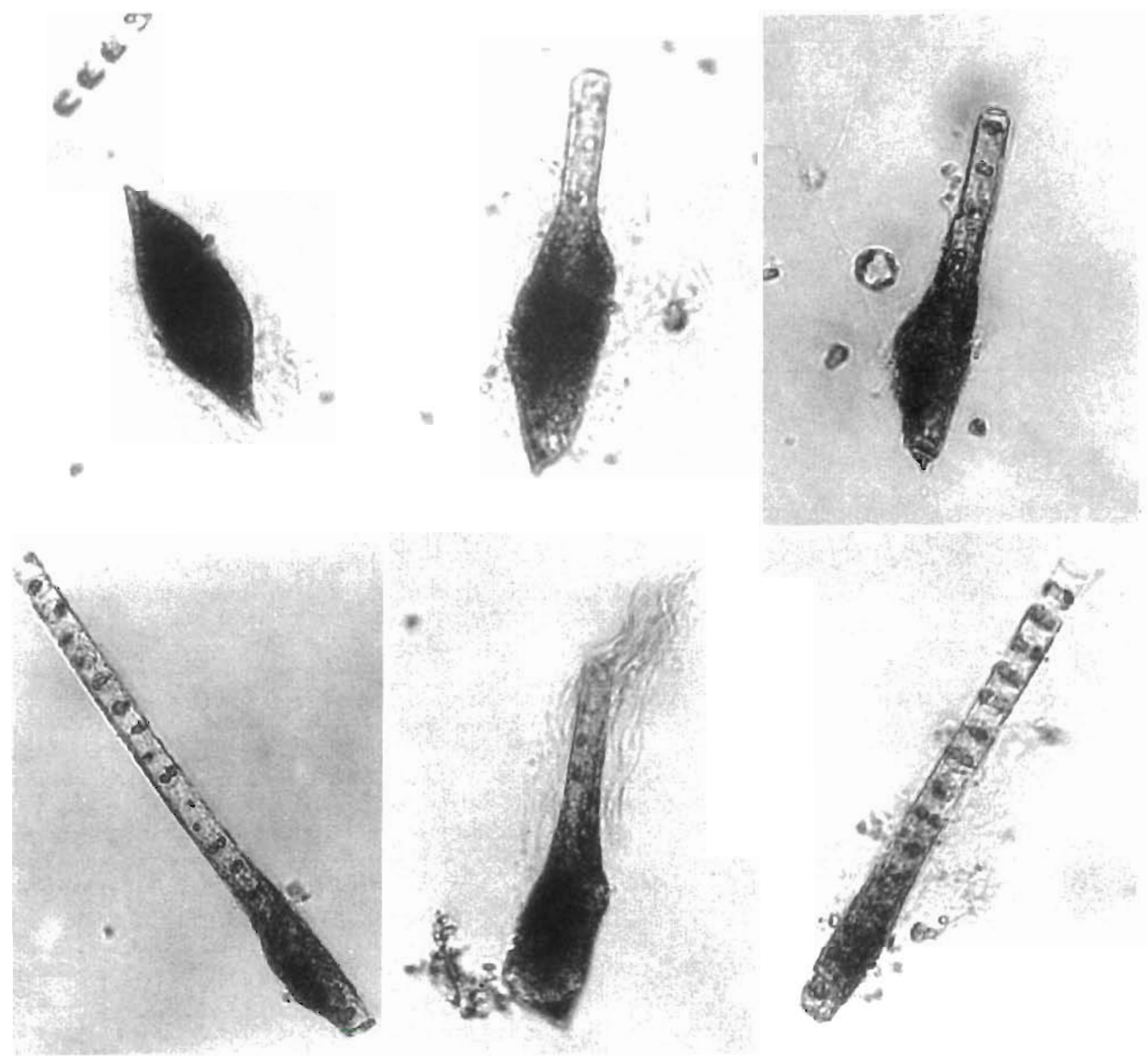

Fig. 8. The heterotrophic dinoflagellate Gyrodinium sp. (upper left panel) and individual Gyrodinium ingesting chain diatoms (all other panels). Samples from Stn 3, 1 m (October)

The implications of this grazing pathway for the cycling of materials in coastal waters are significant. Gyrodinium and related dinoflagellates produce fecal pellets (Nothig \& von Bodungen 1989, Buck et al. 1990. Elbrachter 1991, Buck \& Newton 1995) which can sink to underlying sediments. Unlike the fecal pellets of mesozooplankton grazers, their organic content appears to be negligible (Buck \& Newton 1995), thus uncoupling the flux of silicate from that of carbon and other nutrients. Controls on diatom bloom formation may also be tighter when protists are a significant component of the grazer community. Because protist popu- lation growth rates can be high, protist populations have the capacity to increase rapidly in response to enhanced phytoplankton production (Banse 1992, Frost 1993). At the Stn $3(1 \mathrm{~m})$ site described here, high net growth rates of Gymnodinium $\left(0.6 \mathrm{~d}^{-1}\right)$ and Protoperidinium + Oblea $\left(1.4 \mathrm{~d}^{-1}\right)$ were observed. Rapid grazer population increases can moderate the size of phytoplankton blooms in nearshore waters, influencing the spatial and temporal deposition patterns of bloomderived materials.

In contrast to our findings, Fahnenstiel et aI. (1995) reported no significant microzooplankton grazing on 
diatoms during a summer cruise in the same region. Microzooplankton were observed to graze exclusively on phytoplankton $<20 \mu \mathrm{m}$. The nature of the coupling between producers and consumers is likely to be variable in this spatially patchy and dynamic coastal region. Grazing relationships in natural (unenclosed) waters may be further modulated by predation on microzooplankton by copepods, gelatinous zooplankton, and larval fish (reviewed by Stoecker \& Capuzzo 1990). The copepod Acartia tonsa was shown to consume ciliates in northern Gulf of Mexico bay waters (Gifford \& Dagg 1988), but in general the quantitative significance of such trophic pathways for the prey population is not well known.

High net microzooplankton growth rates were observed during some of our experiments. While Gifford (1988) suggested that the addition of nutrients to dilution experiments may have deleterious effects on oligotrich ciliates, no data are presented and the idea is not substantiated by the sometimes high net growth rates reported here. Net growth rates in higher irradiance incubations $\left(0.8 \mathrm{~d}^{-1}\right)$ were significantly higher than rates in lower irradiance incubations $\left(0.2 \mathrm{~d}^{-1}\right)$. It seems clear that the microzooplankton community was responding to increased phytoplankton growth rates under higher irradiance conditions. While phytoplankton and microzooplankton biomass were closely correlated across all experiments, there were no analogous simple relationships between microzooplankton growth rate and phytoplankton biomass, growth rate or production. Such relationships might be obscured by variations in species composition and trophic interaction across the range of environmental conditions reported here.

Use of the 3-point method should allow a more accurate estimation of growth and grazing rates under conditions of saturated grazing (Gallegos 1989). When net growth of microzooplankton is high in undiluted waters, the most dilute treatments may also be least susceptible to biases caused by changes in microzooplankton density over time. As used here, however, the method suffers from a loss of precision due to reduced numbers of observations (relative to linear-regressionbased estimates) and high natural variability (Evans \& Paranjape 1992). Intended low dilution factors of 0.07 and 0.15 were not always realized when initial chlorophyll concentrations were measured. Lowest dilutions, as well as undiluted treatments, need to be highly replicated for the 3-point method to give meaningful results, especially in comparable nearshore environments where spatial variability is high.

In summary, microzooplankton were found to have a significant grazing impact on phytoplankton during 2 cruises in the continental shelf region of the northern Gulf of Mexico. That impact was sometimes greatest on the larger phytoplankton, and ingestion of chain diatoms by the dinoflagellate Gyrodinium sp. was observed in preserved samples. These observations corroborate the idea that microzooplankton can be an important source of phytoplankton mortality in coastal ecosystems. Further, they suggest that size-based models of trophic interaction within the microplankton may be inappropriate. Because of the diversity of feeding mechanisms among the microzooplankton, their grazing impacts can be as large in eutrophic waters dominated by large phytoplankton cells as in more oligotrophic regions of the ocean.

Acknowledgements. We thank M. Dagg, S. Fick, J. Lynch, $\mathrm{R}$. Toon, and the captain and crew of the RV 'Pelican' for their assistance on the cruises. Irradiance data were provided by S. Lohrenz and D. Redalje. B. Bingham assisted with photography. This research was conducted as part of the Department of Energy Ocean Margins Program, and was funded by grant no. DE-FGO2-92ER61414.

\section{LITERATURE CITED}

Banse K (1982) Cell volumes, maximal growth rates of unicellular algae and ciliates, and the role of ciliates in the marine pelagial. Limnol Oceanogr 27:1059-1071

Banse K (1992) Grazing, temporal changes of phytoplankton concentrations, and the microbial loop in the open sea. In: Falkowski PG, Woodhead AD (eds) Primary productivity and biogeochemical cycles in the sea. Plenum, New York, p 409-440

Biscaye PE, Flagg CN, Falkowski PG (1994) The shelf edge exchange processes experiment, SEEP-II: an introduction to hypotheses, results and conclusions. Deep Sea Res Part II Top Stud Oceanogr 41:231-252

Buck KR, Bolt PA, Garrison DL (1990) Phagotrophy and fecal pellet production by an athecate dinoflagellate in Antarctic sea ice. Mar Ecol Prog Ser 60:75-84

Buck KR, Newton J (1995) Fecal pellet flux in Dabob Bay during a diatom bloom: contribution of microzooplankton. Limnol Oceanogr 40:306-315

Burkill PH, Mantoura RFC, Llewellyn CA, Owens NJP (1987) Microzooplankton grazing and selectivity of phytoplankton in coastal waters. Mar Biol 93:581-590

Bursa AS (1961) The annual oceanographic cycle at Igloolik in the Canadian Arctic. II. The phytoplankton. J Fish Res Bd Can 18:563-615

Chin-Leo G, Benner R (1992) Enhanced bacterioplankton production and respiration at intermediate salinities in the Mississippi River plume. Mar Ecol Prog Ser 87:87-103

Dagg MJ (1995). Ingestion of phytoplankton by the microand mesozooplankton communities in a productive subtropical estuary. J Plankton Res 17:845-857

Dagg MJ, Whitledge TE (1991) Concentrations of copepod nauplii associated with the nutrient-rich plume of the Mississippi River. Cont Shelf Res 11:1409-1423

Elbrachter M (1991) Faeces production by dinoflagellates and other small flagellates. Mar microb Food Webs 5:189-204

Evans GT, Paranjape MA (1992) Precision of estimates of phytoplankton growth and microzooplankton grazing when the functional response of grazers may be nonlinear. Mar Ecol Prog Ser 80:285-290 
Fahnenstiel GL, McCormick MJ, Lang GA, Redalje DG, Lohrenz SE, Markowits M, Wagoner B, Carrick HJ (1995) Taxon-specific growth and loss rates for dominant phytoplankton populations from the northern Gulf of Mexico. Mar Ecol Prog Ser 117:229-239

Frost BW (1972) Effects of size and concentration of food particles on the feeding behavior of the marine planktonic copepod Calanus pacificus. Limnol Oceanogr 17:805-815

Frost BW (1993) A modelling study of processes regulating plankton standing stock and production in the open subarctic Pacific Ocean. Prog Oceanogr 32:17-56

Furnas MJ (1990) In situ growth rates of marine phytoplanton approaches to measurement, community and species growth rates. J Plankton Res 12:1117-1151

Gallegos CL (1989) Microzooplankton grazing on phytoplankton in the Rhode River, Maryland: nonlinear feeding kinetics. Mar Ecol Prog Ser 57:23-33

Gifford DJ (1988) Impact of grazing by microzooplankton in the northwest arm of Halifax Harbour, Nova Scotia. Mar Ecol Prog Ser 47:249-258

Gifford DJ, Dagg MJ (1988) Feeding of the estuarine copepod Acartia tonsa Dana: carnivory vs herbivory in natural microplankton assemblages. Bull mar Sci 43:458-468

Hansen PJ (1992) Prey size selection, feeding rates and growth dynamics of heterotrophic dinoflagellates with special emphasis of Gyrodinium spirale. Mar Biol 114: $327-334$

Jackson GA, Eldridge PM (1992) Food web analysis of a planktonic system off southern California. Prog Oceanogr 30:223-251

Jacobson DM, Anderson DM (1986) Thecate heterotrophic dinoflagellates: feeding behavior and mechanisms. J Phycol $22: 249-258$

Landry MR, Haas LW, Fagerness VL (1984) Dynamics of microbial plankton communities: experiments in Kaneohe Bay, Hawaii. Mar Ecol Prog Ser 16:127-133

Landry MR, Hassett RP (1982) Estimating the grazing impact of marine micro-zooplankton. Mar Biol 67:283-288

Landry MR, Kirshtein J, Constantinou J (1995) A refined dilution technique for measuring the community grazing impact of microzooplankton, with experimental tests in the central equatorial Pacific. Mar Ecol Prog Ser 120: $53-63$

Lessard EJ (1991) The trophic role of heterotrophic dinoflagellates in diverse marine environments. Mar microb Food Webs 5:49-58

Lohrenz SE, Dagg MJ, Whitledge TE (1990) Enhanced primary production at the plume/oceanic interface of the Mississippi River. Cont Shelf Res 10:639-664

This article was presented by D. A. Caron (Senior Editorial Advisor), Woods Hole, Massachusetts, US.A
Longhurst AR (1991) Role of the marine biosphere in the global carbon cycle. Limnol Oceanogr 36:1507-1526

McManus GB, Ederington-Cantrell MC (1992) Phytoplankton pigments and growth rates, and microzooplankton grazing in a large temperate estuary. Mar Ecol Prog Ser 87 . $77-85$

Moloney CL, Field JG (1991) The size-based dynamics pf plankton food webs. I. A simulation model of carbon and nitrogen flows. J Plankton Res 13:1003-1038

Nothig EM, von Bodungen B (1989) Occurrence and vertical flux of faecal pellets of probably protozoan origin in the southeastern Weddell Sea (Antarctica). Mar Ecol Prog Ser 56:281 - 289

Paranjape MA (1987) Grazing by microzooplankton in the eastern Canadian Arctic in summer 1983. Mar Ecol Prog Ser 40:239-246

Putt M. Stoecker DK (1989) An experimentally determined carbonvolume ratio for marine 'oligotrichous' ciliates from estuarine and coastal waters. Limnol Oceanogr 34: $1097-1103$

Riley GA (1937) The significance of the Mississippi River drainage for biological conditions in the northern Gulf of Mexico. J mar Res 1:60-74

Roff JC, Hopcroft RR (1986) High precision microcomputer based measuring system for ecological research. Can J Fish Aquat Sci 43:2044-2048

Sherr EB, Caron DA, Sherr BF (1992) Staining of heterotrophic protists for visualization via epifluorescence microscopy. In: Kemp PF, Sherr BF, Sherr EB, Cole JJ (eds) Current methods in aquatic microbial ecology. Lewis PubIishers, Boca Raton, p 213-227

Sherr EB, Sherr BF, McDaniel J (1991) Clearance rates of $<6 \mu \mathrm{m}$ fluorescently labeled algae (FLA) by estuarine protozoa grazing impact of flagellates and ciliates. Mar Ecol Prog Ser 69:81-92

Stoecker DK, Capuzzo JM (1990) Predation on protozoa: its importance to zooplankton. J Plankton Res 12:891-908

Strathmann RR (1967) Estimating the organic carbon content of phytoplankton from cell volume or plasma volume. Limnol Oceanogr 12:411-418

Strom SL, Buskey EJ (1993) Feeding, growth, and behavior of the thecate heterotrophic dinoflagellate Oblea rotunda. Limnol Oceanogr 38:965-977

Turner RE, Rabalais NN (1991) Changes in Mississippi River water quality this century. BioSci 41:140-147

Welschmeyer N, Goericke R, Strom S, Peterson W (1991) Phytoplankton growth and herbivory in the subarctic Pacific: a chemotaxonomic analysis. Limnol Oceanogr 36: $1631-1649$

Manuscript first received: December 23, 1994

Revised version accepted: August 15, 1995 